\title{
Step Evolution: Improving the Performance of Open-Ended Evolution Simulations
}

\author{
Tiago Baptista \\ CISUC, University of Coimbra \\ Coimbra, Portugal \\ Email: baptista@dei.uc.pt
}

\author{
Ernesto Costa \\ CISUC, University of Coimbra \\ Coimbra, Portugal \\ Email: ernesto@dei.uc.pt
}

\begin{abstract}
A common issue in Artificial Life research, and mainly in open-ended evolution simulations, is that of defining the bootstrap conditions of the simulations. One usual technique employed is the random initialization of individuals at the start of each simulation. However, by using this initialization method, we force the evolutionary process to always start from scratch, and thus require more time to accomplish the objective. Artificial Life simulations, being typically, very time consuming, suffer particularly when applying this method. In this paper, we describe a technique we call step evolution, that can be used to shorten the time needed to evolve complex behaviors in open-ended evolutionary simulations. We provide results from experiments done on an open-ended evolution of foraging scenario, where agents evolve, adapting to a world with a day and night cycle. The results show that the employment of this technique can improve both the overall success of simulation runs, and the time needed to evolve the observed behaviors.
\end{abstract}

\section{INTRODUCTION}

In most Artificial Life research, populations of artificial organisms are evolved throughout several generations. An issue that needs attention when first designing these systems is the creation of the seed population. The choice on how to generate this initial population will have, naturally, a considerable effect on the evolutionary process.

One usual solution to this issue is to randomly initialize the seed population [1] [2]. This solution has, however, at least one drawback. As the initial population of organisms is always randomly initialized on every simulation run, the evolutionary process will always have to start from scratch. These systems are typically very time consuming, having simulation runs easily last several hours to several days or weeks. The random initialization of the seed population will only add to this time requirement.

Another usual solution to the issue is to initialize the seed population with hand-crafted simple organisms [3] [4]. Again, this technique is not without its disadvantages. Although it may shorten the time needed to evolve the population, the definition of an initial seed organism will place the burden of choosing a known good solution to the problem at hand on the researcher, instead of leaving it to evolution to find that solution. To some extent, this technique defeats some of the founding principles of Artificial Life.

One specific area of Artificial Life research where the issue of the seed population is particularly important is in open-

978-1-4673-5863-7/13/\$31.00 (C) 2013 IEEE ended evolution. Although a formal definition of open-ended evolution has not yet gathered consensus, it can be broadly defined as the ability of an evolutionary system to continually produce novel forms [5]. This concept has been considered one of the open issues in artificial life research [6].

To achieve open-ended evolution, a number of authors consider that a major requirement is the absence of an explicitly defined goal or notion of "better" individual [7]. In most evolutionary computation and artificial life research, this notion of "better" individual is implemented via fitness functions. However, in the real world, there are clearly no explicit fitness functions governing the evolution of organisms. In the work described here, we look into the issue of open-ended evolution, considering that simulations don't employ fitness functions or any other kind of artificial selection mechanism.

The absence of an explicitly defined fitness function can generally remove the burden of designing it from the researcher, and thus probably ease its task. For example, in the simulation of complex systems, the design of a fitness function can sometimes be an especially hard issue to tackle. On the other hand, this absence also generally makes the evolutionary progress of the simulations slower and thus requires more computation time.

The requirement of not enforcing a notion of "better" individual makes the solution of randomly initializing the population, better suited to open-ended evolution, than handcoding the initial organisms. By creating an initial solution to seed the population, be it a known-good or only a viable solution, would introduce into the simulation, even if inadvertently, a bias into what is expected of evolution. Conversely, as openended evolution seems harder to model [8], and heavier on computational resources, randomly initializing the population will show its toll on the time requirements of the simulations [9].

In this paper we will describe a technique we call step evolution, that can be used to shorten the time requirements for open-ended evolution simulations. Using it, we are able to use the solution of randomly initializing the population, whilst reducing the computational effort required. We will start by describing the step evolution conceptual model. Next we will detail the experimental setup used to test the technique, and follow with the results from the experiments. We will end with some conclusions and future work. 


\section{Step Evolution}

The simulations described in this paper were implemented using the BitBang framework [10] [11]. Implementing a modern autonomous agent model [12], this framework has roots in Artificial Life systems and Complexity Science. The simulated world is composed of entities. These can either be inanimate objects which we designate as things, or entities that have reasoning capabilities and power to perceive and affect the world - the agents. Both have traits that characterize them, such as color, size, or energy - the features. The agents communicate with, and change the environment using perceptions and actions, taking decisions using the brain.

In this model, there is no definition of a simulation step, as we will not have any type of centralized control. As such, the simulation is asynchronous. The agents will independently perceive, decide, and act. Moreover, there is no fixed evolutionary mechanism included in the definition of the model, since evolution is implemented as an action. That is accomplished by giving the agents the capability of reproduction. Again, there is no central control bound to the process of reproduction. The agents choose when to reproduce by choosing to execute the reproduction action. In addition, there is no explicit fitness function. The agents die due to lack of resources, predators, age, or any other mechanism implemented in the world. Thus, using this model we can simulate open-ended evolution.

Although the model is agnostic regarding the algorithm used for the agents brains, in the experiments shown in this paper, we used an ordered rule list as the brain of the agents. This algorithm uses a a rule list where each rule is composed of a conjunction of conditions. An example of a rule is shown in listing 1 to illustrate. The decision process is straightforward. The rule list is ordered, and rules a evaluated in order. The first rule that evaluates to true is chosen, and its corresponding action is selected.

\begin{tabular}{l}
\hline Listing 1 Example of a rule. \\
IF energy $<10$ AND reach_resource TRUE \\
THEN eat
\end{tabular}

In our model, to keep the open-ended nature of the simulations, we initialize the populations with randomly generated agents. As referred to in the introduction, this can lead to increased time requirements for the simulations. In an effort to tackle this issue, we implemented a technique we call step evolution. To devise it, we took, as usual, inspiration from natural processes.

In complexity science, it is generally accepted that complex systems can be structured hierarchically, where each system is composed of subsystems, these subsystems are themselves composed of subsystems, and going on until we reach the most basic components, like sub-atomic particles. It can also be considered that emergent properties frequently appear across levels. That is, an emergent property observed at a given level, is the product of interactions of components on the level below.

On another note, in biology, an important theory that gathers some consensus is that evolution is not continuous, but hap- pens mostly in large transitions [13]. Smith and Szathmáry's theory states that, although small changes can occur and propagate in a population, the big changes are the ones that drive the evolutionary process.

We combine aspects from the two aforementioned theories to create the step evolution technique. Essentially, it consists of identifying the perceived complexity levels, and dividing the problem accordingly. We first simulate a scaled down version of the problem, and when the agents evolve to solve this simpler problem, we change the simulation by adding the features removed earlier, and continue the evolution. In other words, if a problem with a very large search space, has an extremely small number of possible solutions, we can simplify the problem, eventually maintaining the search space, but increasing the number of solutions. When the simpler version of the problem is solved, we then try to solve the initial problem, starting from the solutions already found.

Obviously, not all problems are prone to this kind of decomposition. For example, when using a standard genetic algorithm to optimize a function, it may not be easy, or possible, to decomposed that function into simpler components. Nonetheless, we believe that evolutionary processes, especially when open-ended, are ideal candidates to apply this technique. In fact, we can find some examples of similar techniques being applied to evolutionary computation, as is the case with the use of incremental fitness functions in evolutionary robotics [14], to some extent in cartesian genetic programming [15], and in an application of genetic algorithms to multiprocessor scheduling [16].

This concept of decomposing a given problem into simple sub-problems has been the focus of study in several areas within artificial intelligence. One such example is the layered learning machine learning technique [17] [18], having been used mainly in robotics, and more specifically in robotic soccer. In this technique, the same principals of task decomposition, and of relating a low-level task learnt with the next level task, are also used. However, in our work we are mainly interested in evolutionary scenarios, and in layered learning works no evolution - as in evolutionary computation-is employed.

In our model, this technique consists of allowing the agents to evolve in steps, by gradually building up the environment and/or the agents' structure (perceptions, actions, features). For example, if an agent is in an environment where the possible survival behaviors are very few, the evolutionary search process will effectively be almost like a random search. We thus, initially setup the environment in such a way that widens the space of viable behaviors, for example by removing constraints. We then let the agents evolve in this simpler environment, and later reintroduce the removed constraints. The agents will then continue to evolve in the new environment, but now from solutions that were already viable in the previous environment. 


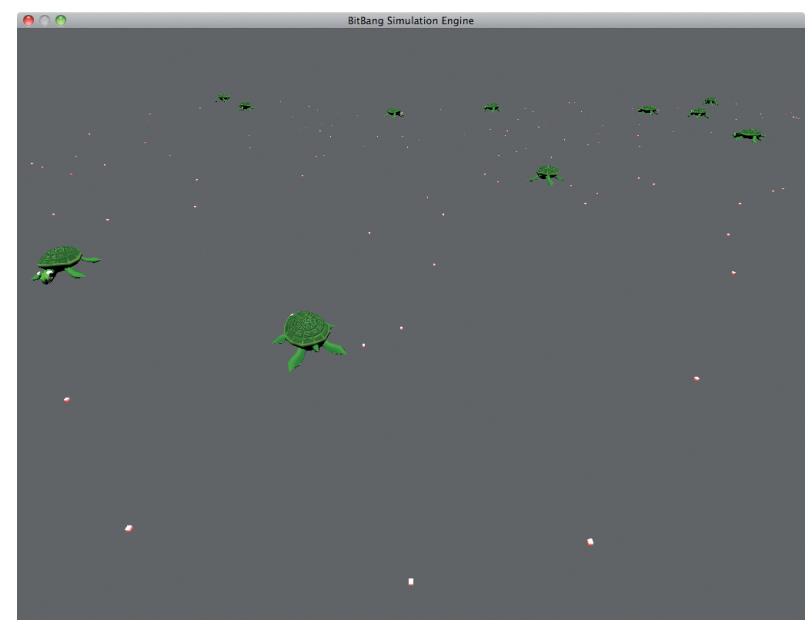

Fig. 1. Screenshot of a running simulation. We can see the agents (turtles), and the edible resources (small cubes).

\section{EXPERIMENTAL SETUP}

To test the step evolution technique, we implemented a world where agents need to evolve foraging capabilities and adapt to an environment with day and night. We follow the same experimental setup as the one from a previous openended evolution experiment described in [19].

In those simulations we implement a world where both agents and resource items are placed. To survive, the agents need to search for and eat the resource items. The terrain is a square. This area restricts the placement of agents, and resources, but does not restrict the movement of the agents. The world is infinite, i.e., an agent can move past the boundaries of the populated terrain. At startup, the field is populated with a configured amount of randomly placed food items. These are periodically replenished so that the total food count is maintained.

To differentiate the day from the night, the environment has a light level that oscillates between a configurable maximum and minimum. For each day the maximum light level is randomly calculated as the overall maximum minus a random value between zero and the delta. The same applies for the day's minimum. For example, if the maximum light level is 100 , the minimum light level is 0 , and the delta is 10 , each day's maximum light level will be a random value between 90 and 100, and each day's minimum light level will be a random value between 0 and 10 . Additionally, the light level does not rise or fall abruptly, but rather changes linearly during a specified time interval, simulating dusk and dawn.

In this environment, the agents will need to evolve the capabilities to find and eat food, to reproduce, and to synchronize with the day and night cycle, sleeping during the night, and moving and eating during the day.

The agents have a set of features, perceptions and actions, that define their structure. In this world the agents have the following structure:

- Features:
- Energy: This feature represents the current energy level of the agent. When this feature reaches zero, the agent dies. The feature is initialized with a predetermined value at agent birth.

- Metabolic Rate: The metabolic rate is the amount of energy the agent consumes per time unit. This rate is initialized to its configured base value, and changes as the agent moves (increases) or sleeps (decreases).

- Birth Date: This feature is set to the current time at birth. It is used to calculate the agent's age. When the agent reaches a given age, it dies. This procedure allows the evolution to continue past the moment when the agents have developed good navigation and eating capabilities, whilst maintaining an open-ended simulation.

- Perceptions:

- Energy: This is a self-referencing perception on the agent's current energy level. This perception is tied to the corresponding feature.

- Resource Location: This is the agent's perception of vision, representing the position of the nearest resource, relative to the agent's position and orientation. The agent's vision field is defined by a given range and angle. An object is within the vision field of an agent if its distance to the agent is less than or equal to the vision range, and the relative angle to the agent is within the defined vision angle. This is a numerical perception with possible values $0,1,2$, and 3 . The value 0 means no resource is visible, 1 means there is a resource to the left, 2 means there is a resource directly in front of the agent, and 3 means there is a resource to the right. This perception is influenced by the light level of the environment. As the light level drops, so does the range of vision for the agent.

- Reach Resource: This is a boolean perception that evaluates to true whenever the agent has a resource within its reach.

- Light Level: This perception gives the agent the power of sensing the brightness of the environment. This can also be considered a perception of vision. The value of the perception is numeric and, at each time, is evaluated to the environment's current light level.

- Actions:

- Movement: We define three actions for movement. One to walk forward, one to turn left, and one to turn right.

- Eat: This action enables the agent to eat a resource within its reach. If no resource is within reach when the action is executed, nothing happens. This action will add a configured amount of energy to the agent's energy feature.

- Sleep: The agent can use this action to sleep. In this simulation, when an agent is sleeping, it will 
stand still and its metabolic rate will decrease, falling below the base metabolic rate and thus allowing the agent to conserve energy.

- Reproduce: This action allows the agent to reproduce itself. The reproduction implemented is asexual. When the action is executed, a new agent is created and placed in the world. The new agent will be given a brain that is a mutated version of its parent's brain. The action will also transfer energy from the parent to the offspring. The amount of energy consumed in the action is the sum of the initial energy for the new agent and a configurable fixed cost for the action.

The algorithm used as a brain for the agents is an ordered rule list, as described in the previous section, and the same used in [19].

As mentioned before, we randomly initialize the population when we start the simulation. That means that initially the agents will most likely not be able to search for food, eat, or even reproduce. To prevent the population from dying out right from the start of the simulation, we keep introducing new agents into the population until they evolve to be selfsustained, i.e. when they are capable of searching for food, eating, and reproducing. When the global number of agents falls bellow a given threshold, we randomly pick an agent from the live ones, and force the reproduction of that agent. If there are no live agents, a completely random new one is generated. Once the population can self-sustain, no new agents are forcedly introduced.

To implement the step evolution technique in this world, we setup two different options. First, we implemented a version where we remove the light level variation from the environment for the first step, and then reintroduce it later in the simulation, as a second step. This way, the evolution of foraging will happen in an environment that is less challenging. The other option implemented was to also scale down the agent structure for the first step of the simulation. In this case, we removed from the agent the perception of the light level, and the action of sleeping, re-adding them on the second step of the simulation. With these two variants we intend to apply the step evolution concept to the environment and to the structure of the agents.

Simulations were configured following previous experimentation on the same scenario [19], but without step evolution. Here, we are mainly interested in the time it takes to evolve behaviors adapted to this environment, and with the parameters that are specific to the step evolution technique. We thus created several experiments with different configuration values. For each experiment we ran thirty independent simulations. The parameters that we tested here were the time limit of the runs, and the time at which the second step starts. In addition, we also divided the experiments into two groups. First, we tested only environmental step evolution, and then we tested both environmental and structural step evolution. In table I we show the various experimental configurations tested.

To analyze the results from the various parameter configuration variations, we determined the statistical significance of
TABLE I

CONFIGURATION VALUES FOR THE SEVERAL EXPERIMENTS RAN.

\begin{tabular}{lrr}
\hline Step Evolution & Time Limit & Change Time \\
\hline \multirow{2}{*}{ None } & $100 \mathrm{k}$ & 0 \\
& $200 \mathrm{k}$ & 0 \\
\hline & & $20 \mathrm{k}$ \\
Environmental & $100 \mathrm{k}$ & $50 \mathrm{k}$ \\
& & $70 \mathrm{k}$ \\
\cline { 2 - 3 } & $200 \mathrm{k}$ & $50 \mathrm{k}$ \\
& & $100 \mathrm{k}$ \\
\hline & & $20 \mathrm{k}$ \\
& $100 \mathrm{k}$ & $50 \mathrm{k}$ \\
Environmental + Structural & & $70 \mathrm{k}$ \\
\cline { 2 - 3 } & $200 \mathrm{k}$ & $50 \mathrm{k}$ \\
& & $100 \mathrm{k}$ \\
\hline
\end{tabular}

the null hypothesis of no difference for each set of experiments using Kruskal-Wallis ANOVAs with $\alpha=0.05$. If a significant difference in one of these sets of experiments was found, further pairwise Mann-Whitney U tests with Holm's p-value adjustment were conducted. These non-parametric tests were chosen because the data is not guaranteed to follow a normal distribution, and runs are independent and non-related.

\section{RESUlTS}

In this section we show and analyze the results from the experiments conducted. As we are mainly interested in finding if the technique allows for lower time requirements, we look at the time when important behavioral features emerge in the population. Namely, we will analyze the time when agents start having a good foraging behavior, the time when the population starts to have self-sustained reproduction behaviors, and the time when the population adapts to the daily cycle, sleeping during the night, and being active during the day. To better illustrate, we show in Fig. 2 an evolutionary plot from a typical run of an experiment without step evolution. We can clearly pinpoint the time when the agents start gathering food (about 16000 ), the time when they start to reproduce (about 34000 ), and the time when they synchronize (about 36000 ).

Although it is easy to pinpoint the time at which these behaviors emerge by analyzing the evolutionary plots, we needed to do it programmatically. To that end, scripts were created to analyze the simulation logs, and determine these performance metrics. To determine both the time for evolution of foraging and for evolution of synchronization, we go through the whole simulation, starting from the end of the simulation to the beginning, calculating the average of all agents, alive at that time, for the given metric. When this average drops below a given threshold, that is the time when the behavior evolved. The thresholds used for these experiments where 6 for the gathered energy, and 0.3 for the ratio of agents in sync over all agents with known sync. To determine the time when the population starts to have self-sustained reproduction, we again go through the simulation starting from the end, and find the last agent that was forcibly reproduced. 


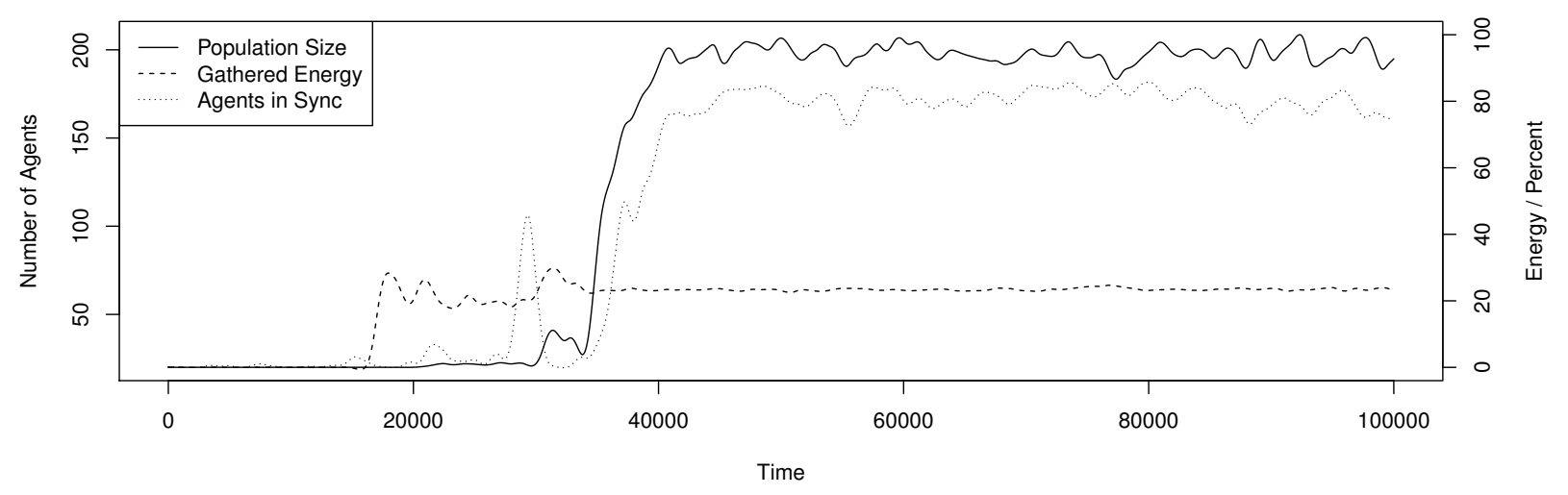

Fig. 2. Plot of the evolution of the number of agents, their average gathered energy, and percentage of agents in sync, over the course of one simulation run.
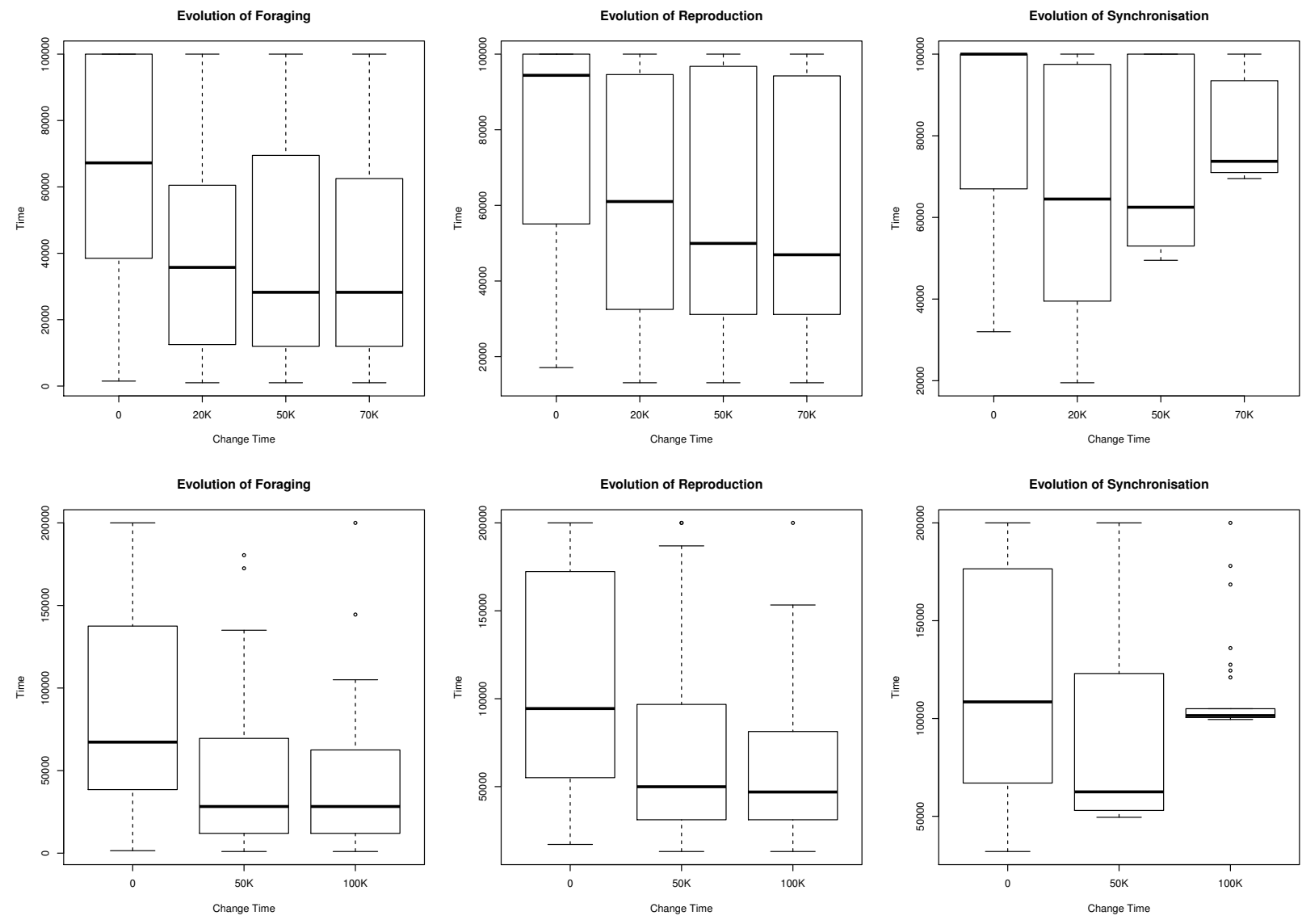

Fig. 3. Results from the experiments with a time limit of 100k (top) and of 200k (bottom), using only environmental step evolution.

First, we ran the experiments that test the application of step evolution to the environment. As mentioned earlier, we tested both for the 100k time limit and 200k time limit. In fig 3 we compare the results from these two sets of experiments. Examining the boxplots in this figure, we can see that there seems to be an improvement when using step evolution, regarding all tested performance values. In fact, performing the statistical tests, we found that there are significant differences in the results.
Considering the results from fig 3 , we found that in all the performance values there are significant differences in the results, with a p-value of 0.013 for the evolution of foraging, 0.015 for the evolution of reproduction, and 0.013 for the evolution of synchronization. Further pairwise tests revealed that, regarding the evolution of foraging, all step evolution configuration values $(20 \mathrm{k}, 50 \mathrm{k}$, and $70 \mathrm{k})$, perform better than the experiment without step evolution. However, when looking at the evolution of reproduction or synchronization, pairwise 

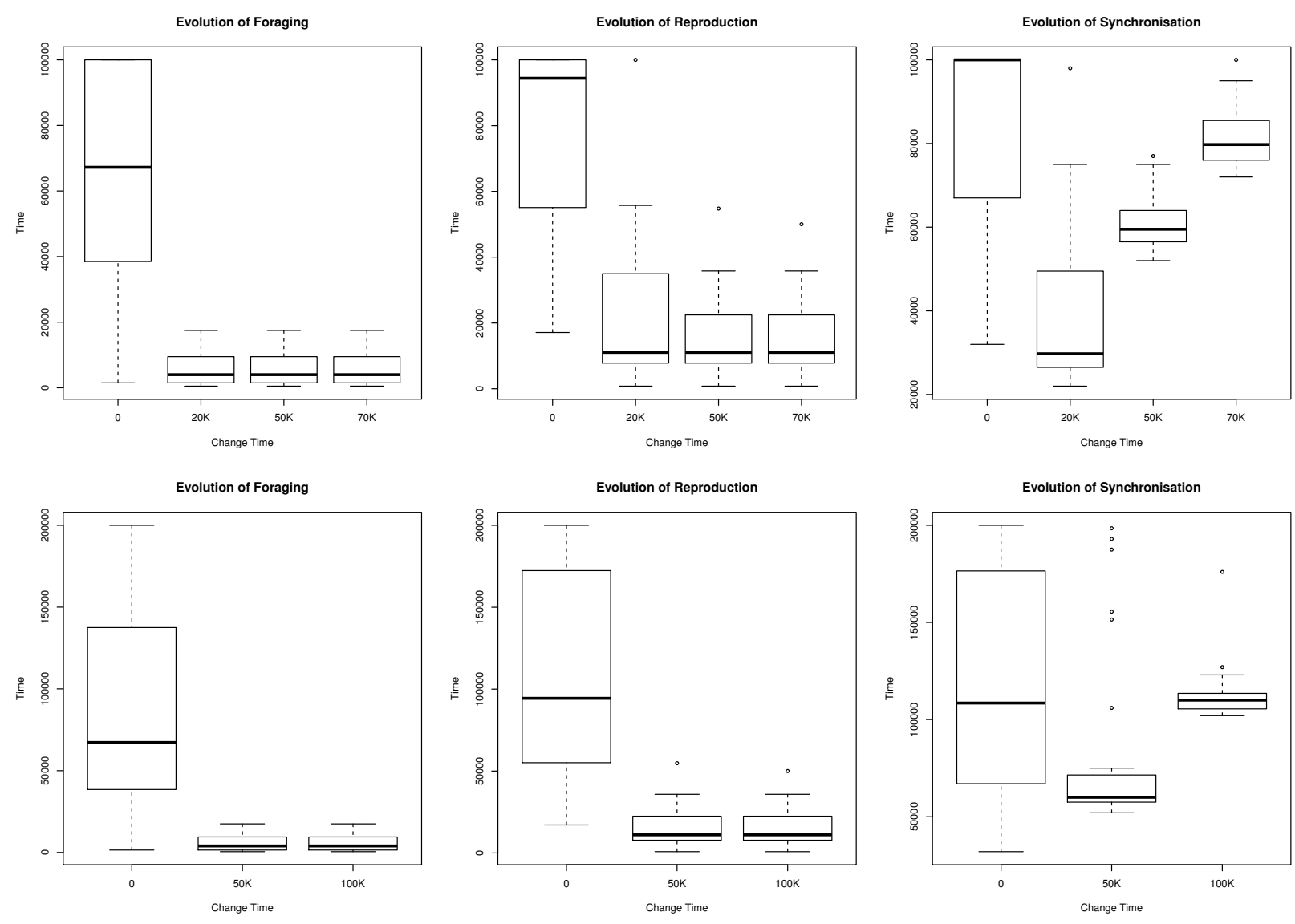

Fig. 4. Results from the experiments with a time limit of 100k (top) and of 200k (bottom) and using both environmental and agents' structure step evolution.

tests reveal that we can only find a significant difference in some of the experiments. Namely, in the evolution of reproduction, only the $50 \mathrm{k}$ and $70 \mathrm{k}$ configurations perform better than the configuration without step evolution, and in the evolution of synchronization, only the 20k performs better than the configuration without step evolution.

Regarding the experiments with a time limit of 200k, we find similar results. We found there are significant differences in the results for all the performance values, with p-values of about $0.004,0.002$, and 0.034 . Further pairwise tests, again, revealed that in al configurations (50k and 100k), the experiments with step evolution perform significantly better than the experiment without step evolution for the evolution of foraging and reproduction. However, in the case of the evolution of synchronization, we didn't find significant differences in using step evolution. We only found that when using step evolution, the configuration of $50 \mathrm{k}$ performs significantly better than 100k configuration. This result can be due to the fact that in most runs, agents could adapt to the daily cycle before the change of environment that only happens at time 100k. In fact, the boxplot shows that in most runs the agents synchronize soon after the change.

We then ran the experiments where we use step evolution on both the environment and on the structure of the agents.
TABLE II

PAIRWISE TESTS FOR THE EVOLUTION OF SYNCHRONIZATION FOR THE EXPERIMENTS WITH 100K TIME LIMIT, AND USING BOTH ENVIRONMENTAL AND STRUCTURAL STEP EVOLUTION.

\begin{tabular}{l|lll} 
& 0 & $20 \mathrm{k}$ & $50 \mathrm{k}$ \\
\hline $20 \mathrm{k}$ & $1.43 \times 10^{-8}$ & - & - \\
$50 \mathrm{k}$ & 0.0003 & $9.68 \times 10^{-9}$ & - \\
$70 \mathrm{k}$ & 0.0610 & $5.60 \times 10^{-12}$ & $5.15 \times 10^{-14}$
\end{tabular}

We show the results from those experiments in Fig.4 for the experiments with $100 \mathrm{k}$ and 200k time limit. Analyzing the boxplots, we get a clear indication that the use of step evolution greatly improves the time requirements to evolve foraging, reproduction, and synchronization to the daily cycle. The statistical tests support this analysis.

On the experiments with 100k time limit, we find significant differences in the results for all the analyzed values. In those experiments, we find a p-value of $8.943 \times 10^{-12}$ for the evolution of foraging, a p-value of $1.566 \times 10^{-12}$ for the evolution of reproduction, and a p-value of $1.446 \times 10^{-15}$ for the evolution of synchronization. For the experiments with 200k time limit we find p-values of $4.869 \times 10^{-11}, 1.490 \times 10^{-12}$, and $3.014 \times 10^{-4}$, respectively. Further pairwise tests reveal that, for all the performance values, all but one of the experiments 
TABLE III

NUMBER OF SUCCESSFUL RUNS FOR EACH EXPERIMENTAL CONFIGURATION.

\begin{tabular}{|c|c|c|c|c|c|}
\hline Step Evolution & Time Limit & Change Time & Runs & Foraging & Sync \\
\hline \multirow{2}{*}{ None } & $100 \mathrm{k}$ & 0 & 30 & 16 & 13 \\
\hline & $200 \mathrm{k}$ & 0 & 30 & 24 & 24 \\
\hline \multirow{5}{*}{ Environmental } & \multirow{3}{*}{$100 \mathrm{k}$} & $20 \mathrm{k}$ & 30 & 23 & 21 \\
\hline & & $50 \mathrm{k}$ & 30 & 23 & 22 \\
\hline & & $70 \mathrm{k}$ & 30 & 24 & 24 \\
\hline & \multirow{2}{*}{$200 \mathrm{k}$} & $50 \mathrm{k}$ & 30 & 28 & 28 \\
\hline & & $100 \mathrm{k}$ & 30 & 29 & 29 \\
\hline \multirow{5}{*}{ Environmental + Structural } & \multirow{3}{*}{$100 \mathrm{k}$} & $20 \mathrm{k}$ & 30 & 29 & 29 \\
\hline & & $50 \mathrm{k}$ & 30 & 30 & 30 \\
\hline & & $70 \mathrm{k}$ & 30 & 30 & 29 \\
\hline & \multirow{2}{*}{$200 \mathrm{k}$} & $50 \mathrm{k}$ & 30 & 30 & 30 \\
\hline & & $100 \mathrm{k}$ & 30 & 30 & 30 \\
\hline
\end{tabular}

with step evolution perform significantly better than the ones without step evolution. In table II we show the pairwise tests performed for the evolution of synchronization, and time limit of $100 \mathrm{k}$. There is only one case here where we were not able to determine that a significant difference exists when comparing to the experiment with and without step evolution (change time of $70 \mathrm{k}$ ). Again, as was the case for the experiments with only environmental step evolution, this indicates that in this case, the change happens too late to effect a significant difference when compared to the experiment without step evolution. The same happens for the configuration of 100k change time, when the time limit is $200 \mathrm{k}$.

Another important performance metric is the number of runs from each experiment that are successful. We say that a run is successful if, in the time limit assigned, the agents evolve the behaviors adapted to the environment and have a self-sustained population. In this case, we are looking to see, from the thirty runs, how many are successful in first evolving foraging and reproduction, but mainly in evolving synchronization. One important thing to note is that, if in a run, the agents don't evolve a foraging behavior, they will obviously not be able to evolve any other behavior. In table III we show these results for all the experiments.

Again, when looking at this performance metric, we can see that using step evolution does allow a higher number of runs to be successful in evolving synchronization. Especially in the experiments with a time limit of $100 \mathrm{k}$, we can go from having 13 successful runs without step evolution, to 30 successful runs with both environmental and structural step evolution. On the experiments with a time limit of 200k, the difference is not as pronounced, but the goal here is to exactly lower the time limit required, and still have a large number of successful runs. The experiments with a time limit of 200k, had already a high number of successful runs before applying the step evolution technique, so the effect on this metric is not as noticeable.

\section{CONCLUSIONS}

Considering the results from all the experiments, it is fair to conclude that the step evolution technique did provide a significant advantage in terms of the time requirements of these open-ended evolution simulations. We have also learned that the practical application of the technique is fairly straightforward in open-ended evolution. In this kind of scenario, as we don't use an explicit fitness function, it is easier to divide the evolutionary process into steps simply by incrementally building up the environment and the agents' structure. We have shown that, for the scenario presented here, when we apply step evolution to both the environmental conditions, and to the structure of the agents, we can successfully use the 100k time limit, and still have a good performance from all runs of the experiment.

In terms of future work, having tested the technique in one simulation scenario, it is now important to also test in other scenarios. In the experiments presented we tested the technique with two steps. A larger number of steps should be tested as well. Also, in the experiments presented in this paper, we tested several values for the configuration parameter that sets the time at which we change the step. It could be possible to automatically detect if the population has evolved the behaviors adapted to the current step, and thus make this change dynamic and adapted to each run. Note however that, as we are not using fitness functions, we cannot simply test the populations fitness. We will need to devise a detection method that doesn't hinder the open-endedness of the simulations.

\section{REFERENCES}

[1] L. Yaeger, "Computational genetics, physiology, metabolism, neural systems, learning, vision, and behavior or Poly World: Life in a new context," in Artificial Life III: Proceedings of the Workshop on Artificial Life, C. G. Langton, Ed. Addison-Wesley, 1994, pp. 263-298.

[2] A. Channon, "Evolutionary Emergence: The Struggle for Existence in Artificial Biota," Ph.D. dissertation, University of Southampton, Nov. 2001.

[3] T. S. Ray, "Evolution, Ecology and Optimization of Digital Organisms," Tech. Rep. 92-08-042, 1992.

[4] A. Eiben, A. R. Griffioen, and E. Haasdijk, "Population-based Adaptive Systems: an Implementation in NEWTIES," in ECCS 2007 - European Conference on Complex Systems, Dresden, Germany, Jul. 2007.

[5] R. K. Standish, "Open-ended artificial evolution," International Journal of Computational Intelligence and Applications, vol. 3, no. 2, pp. 167175, 2003. 
[6] M. A. Bedau, J. S. McCaskill, N. H. Packard, S. Rasmussen, C. Adami, D. G. Green, T. Ikegami, K. Kaneko, and T. S. Ray, "Open problems in artificial life." Artificial Life, vol. 6, no. 4, pp. 363-376, 2000.

[7] A. Channon, "Three evolvability requirements for open-ended evolution," in Artificial Life VII Workshop Proceedings, C. C. Maley and E. Boudreau, Eds., Portland, OR, 2000, pp. 39-40.

[8] C. Maley, "Four steps toward open-ended evolution," GECCO-99: Proceedings of the Genetic and ..., 1999.

[9] T. Baptista and E. Costa, "The Evolution of Foraging in an Open-Ended Simulation Environment," in Progress in Artificial Intelligence, 15th Portuguese Conference on Artificial Intelligence, EPIA 2011, Lisbon, Portugal, October 2011, L. Antunes and H. S. Pinto, Eds. SpringerVerlag, Oct. 2011, pp. 125-137.

[10] T. Baptista, T. Menezes, and E. Costa, "Bitbang: A model and framework for complexity research," in Proceedings of the European Conference on Complex Systems 2006, Oxford, UK, Sep. 2006, p. 73.

[11] T. Baptista, "Complexity and Emergence in Societies of Agents," Ph.D. dissertation, University of Coimbra, Coimbra, Jul. 2012.

[12] S. Russell and P. Norvig, Artificial Intelligence: A Modern Approach (2nd Edition), 2nd ed. Prentice Hall, Dec. 2002.
[13] J. M. Smith and E. Szathmáry, The Major Transitions in Evolution. Oxford: Oxford University Press, 1985.

[14] A. L. Nelson, G. J. Barlow, and L. Doitsidis, "Fitness functions in evolutionary robotics: A survey and analysis," Robotics and Autonomous Systems, vol. 57, no. 4, pp. 345-370, Apr. 2009.

[15] J. F. Miller, Ed., Cartesian Genetic Programming, 1st ed., ser. Natural Computing Series. Springer, Sep. 2011.

[16] A. S. Wu, H. Yu, S. Jin, K.-C. Lin, and G. Schiavone, "An Incremental Genetic Algorithm Approach to Multiprocessor Scheduling," IEEE Transactions on Parallel and Distributed Systems, vol. 15, no. 9, Sep. 2004.

[17] P. Stone, "Layered Learning in Multi-Agent Systems," Ph.D. dissertation, Carnegie Mellon University, 1998.

[18] P. Stone and M. M. Veloso, "Layered Learning," in ECML '00: Proceedings of the 11th European Conference on Machine Learning. SpringerVerlag, May 2000.

[19] T. Baptista and E. Costa, "Evolution of a multi-agent system in a cyclical environment," Theory in Biosciences, vol. 127, no. 2, pp. 141-148, Jun. 2008. 